\title{
Design and Analytical Analysis of a Novel DBA Algorithm with Dual-Polling Tables in EPON
}

\author{
Jiunn-Ru Lai, Hsin-Yi Huang, Wen-Ping Chen, Luke K. Wang, and Ming-Yuan Cho \\ Department of Electrical Engineering, National Kaohsiung University of Applied Sciences, Kaohsiung 807, Taiwan \\ Correspondence should be addressed to Wen-Ping Chen; wpc@kuas.edu.tw
}

Received 29 September 2014; Accepted 26 December 2014

Academic Editor: Mo Li

Copyright ( 2015 Jiunn-Ru Lai et al. This is an open access article distributed under the Creative Commons Attribution License, which permits unrestricted use, distribution, and reproduction in any medium, provided the original work is properly cited.

\begin{abstract}
Ethernet passive optical network is a point-to-multipoint structure, and an effective media access protocol should be designed for collision avoidance and bandwidth allocation. Most previous researches have aimed to solve the problem using dynamic bandwidth allocation (DBA) algorithms with polling-based schemes. Unfortunately, idle channel time among the polling cycles cannot be overcome efficiently. In this paper, a dual-polling DBA (DP-DBA) algorithm which consists of an interpolling mechanism and an intrapolling mechanism is proposed. The interpolling mechanism helps eliminate the idle time problem while the intrapolling part aggregates the unused bandwidth of the light-loaded users and those due to nondefragmentation of the Ethernet frames of the general users. We also evaluate the performance of the DP-DBA algorithm by a comparison with the interleaved polling with adaptive cycle time (IPACT), the dynamic bandwidth allocation with a modified grant table generation algorithm (DBA2), the Double-Phase Polling (DPP), and the adaptive DBA algorithm with sorting report messages (Sort-DBA) schemes with simulation results. For greater realism with regard to general applications, we also simulate the asymmetric traffic loads at the optical network units (ONUs).The results show that DP-DBA outperforms the other DBA schemes under asymmetric load conditions.
\end{abstract}

\section{Introduction}

The number of subscribers served by FTTx equipment is strongly increasing [1]. The ongoing of market is prosperous in different regions of the world such as in Asia [2], the United States, and Latin America [3]. Among broadband access network technologies, the Ethernet passive optical network (EPON) is a promising candidate for future ISP last-mile solutions [4]. EPON has many advantages for a subscriber access network, for example, reduced cost of maintenance and ease of upgrade. EPON also has properties which include wide coverage area, reduced fiber deployment, and both multicast and broadcast capacities. Typically, EPON makes use of three wavelength bands, namely, $1310 \mathrm{~nm}, 1490 \mathrm{~nm}$, and $1550 \mathrm{~nm}$. The $1490 \mathrm{~nm}$ wavelength is responsible for downstream broadcast from the optical line terminal (OLT) to multiple optical network units (ONUs). At the upstream side, the $1310 \mathrm{~nm}$ wavelength is used for the multiple ONUs to transmit the data to the OLT. However, the shared media access property at the upstream side needs a sophisticated media access control (MAC) for the multiple-to-one structure $[5,6]$ in different network environments such as heterogeneous propagation delay [7] and long-reach [8]. Accordingly, the performance of these schemes is evaluated by analysis approach [9] and analysis approach [10]. An arbitrary mechanism is needed to prevent simultaneous transmissions and data collision. Therefore, an interleaved polling scheme, called interleaved polling with adaptive cycle time (IPACT), is proposed to cope with this problem by Kramer et al. [11]. With the limited service discipline, this algorithm achieves good performance. However, the idle time issue is not effectively resolved. Further, due to the busty nature of Ethernet traffic and prohibition of fragmentation of Ethernet packets, some ONUs may have less traffic to transmit while other ONUs may have more traffic to transmit and need more bandwidth in each transmission cycle. Consequently, IPACT allocation of bandwidth based on a single ONU is not globally optimized.

Assi et al. [12] proposed a DBA algorithm which makes use of the excess bandwidth of lightly loaded ONUs to help the heavily loaded ONUs to transport their bandwidth 
demand in each transmission cycle, thus improving the performance of limited allocation schemes. To deal with the idle time issue, they proposed an early allocation mechanism, called DBA2, which schedules lightly loaded ONUs without waiting till end of collection of all REPORT messages. In contrast, it schedules heavily loaded ONUs only after the OLT receives all REPORT messages and performs computation for bandwidth allocation. In addition, a fair-excess scheme is applied to DBA2 to obtain high performance for WDMEPON [13]. Unfortunately, the DBA2 algorithm works well with the idle period only under low or medium traffic loads. Moreover, for high traffic load conditions, ONUs have a bandwidth demand larger than the minimum guaranteed bandwidth, so GATE messages cannot be transmitted early to the ONU for idle time compensation because of not enough number of lightly loaded ONUs. Hence an early DBA mechanism with prediction-based fair excessive bandwidth allocation scheme was proposed in 2008 [14]; however, its prediction scheme cannot correctly estimate the bandwidth requirements. In 2006, Zheng [15] proposed a bandwidth allocation method, which makes use of a tracker value to deal with the idle time problem under high traffic loads. This algorithm improves the DBA2 idle time issue under heavy load conditions. However, due to the redundant overhead of the processing time of the tracker and the regular REPORT messages, bandwidth wastes still exist when the ONU is under heavy load. Further, an unfairness issue arises. It is supposed that when the previous ONUs are operating under a light load condition, the following ONUs can share the remainder bandwidth; but the previous ONUs cannot allot the remainder bandwidth if the following ONUs are operating under a light load. The idle time results from the OLT waiting for the last REPORT from the last ONU during one cycle. In 2009, Choi et al. [16] proposed a DoublePhase Polling (DPP) algorithm to divide N ONUs into two subgroups; these two subgroups will counteract each other's idle time to increase the utilization of upstream transmission. However, the performance of DPP algorithm is similar to IPACT with limited service (IPACT-limited) algorithm in uniform traffic condition.

In 2010, the authors propose an adaptive DBA algorithm with sorting report messages (Sort-DBA) to tackle the problem of idle time [17]. The minimum guaranteed bandwidth $\left(B_{\min }\right)$ is redefined and the last REPORT is sent before data transmission of the last ONU during the cycle time. However, the unused bandwidth of the light load ONUs cannot be reallotted for the heavy load ONUs. In 2011, Chan et al. [18] proposed a Local-Traffic-Redirection-Based Dynamic Bandwidth Assignment algorithm to balance the load assignment. The ONUs are divided into two groups, with each group assigned an Action Remote Repeater Number (Action RN) to identify if the destination node belongs to the same group. The unused bandwidth can be used to transmit the data buffered at the heavy load ONUs. However, active remote repeaters are needed. This increases the node complexity and device's cost. It also violates the passive and low-cost properties of EPON.

In 2005, the authors [19] make use of the Multipoint Control Protocol (MPCP) with threshold reporting and with inter- and intra-ONU priority scheduling to solve the DBA and frame fragmentation problem. However, heavy computation and large table space are needed. In 2014, Sue et al. [20] proposed a nonpredictive-based ONU scheduling method designated as active intra-ONU scheduling with proportional guaranteed bandwidth (ASPFB) to improve the unused slot remainder (USR) problem for EPONs. Multiple queue report variables must be updated in the reports by the ONUs in each polling cycle. It incurs somehow computational loads on ONU. In this paper, we take use of the dual-polling mechanism, which involves only two values. As compared to the previous work, our scheme is more suitable for real implementation. In 2006, Chen et al. [21] proposed an efficient and fine scheduling algorithm for bandwidth allocation. With the ONU's report of the USR value of itself in this round, OLT can arrange the next ONU to start transmission in advance. However, the polling scheme must be the interleaved form. In this paper, our proposed scheme, dual-polling scheme, can be applied to offline or online schemes without any limitation.

There is also a trend for the use of WDM-PON in broadband access networks. However, the DBA algorithm for WDM-PON still needs more researches. This present study proposes a dual-polling DBA (DP-DBA) algorithm for utilization improvement and unused bandwidth reallocation. Section 2 addresses DP-DBA operation. Simulation results are presented in Section 3. This study is concluded with a few relevant remarks and also addresses the direction of our future research.

\section{Dual-Polling DBA Algorithm}

The DP-DBA algorithm consists of two polling schemes which are designated as the interpolling and the intrapolling schemes. Two polling tables are included, Layer I polling table and Layer II polling table. The former is designed to record the bandwidth requests and the round-trip time (RTT) from the ONUs under the minimum bandwidth allocation $\left(B_{\min }\right)$. The latter records the extra bandwidth requests of $\left(B_{\min }\right)$ from the heavy load ONUs. The interpolling scheme solves the idle time problem. To reallot the unused bandwidth from the light load ONUs to the heavy load ONUs, the intrapolling scheme is proposed. The operation of the two schemes will now be addressed.

2.1. Interpolling Scheme. For general DBA algorithms, an OLT cannot start DBA operation for cycle time $(I+1)$ until receiving all the REPORT messages from the ONUs during cycle time $I$. After processing the messages, the upstream bandwidth allocation decision for cycle time $(I+1)$ can be made and broadcasted to the ONUs. However, the idle time period at the upstream side between the last transmission data at cycle time $I$ and the first transmission data at cycle time $(I+1)$ is wasted. Referring to [17], we can express the idle period as

$$
T_{\mathrm{idle}}=T_{\mathrm{dba}}+\mathrm{RTT}+T_{\mathrm{ONU}},
$$




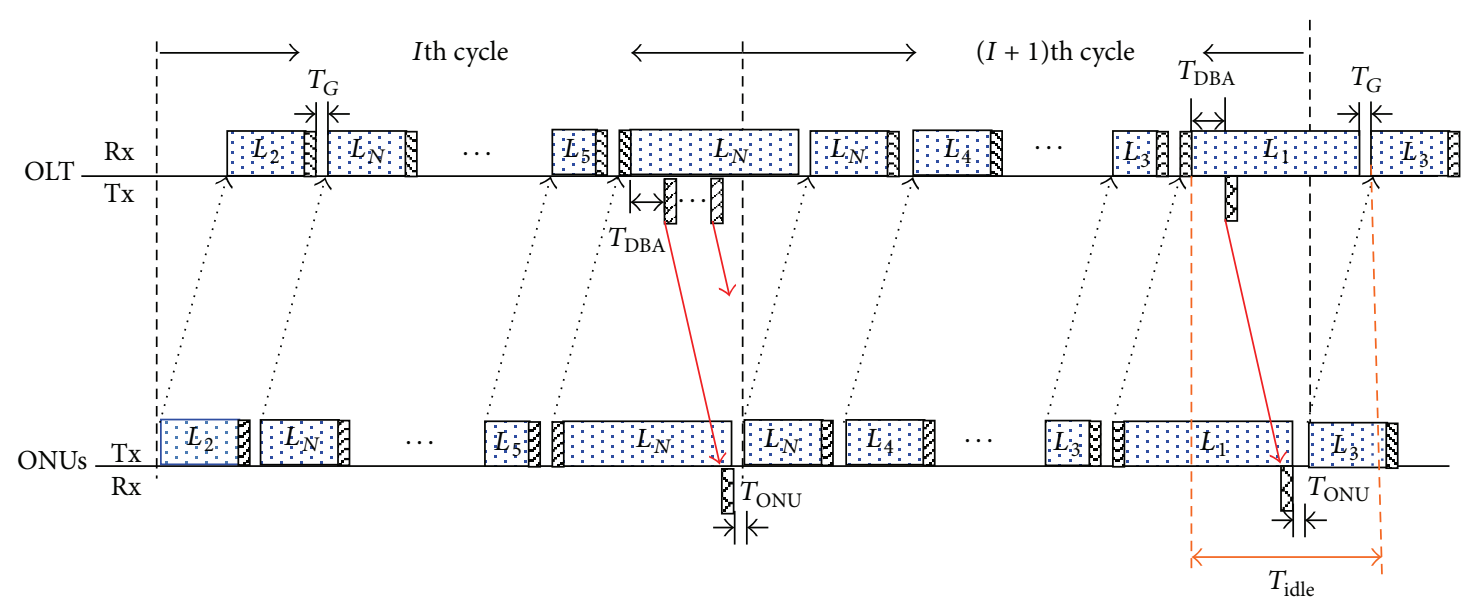

OLT: optical line terminal

ONU: optical network unit

Tx: transmit

$\mathrm{Rx}$ : receive

$T_{\mathrm{ONU}}$ : processing time at $\mathrm{ONU}$

$T_{\text {idle }}$ : idle time

$T_{\mathrm{DBA}}$ : DBA calculation time at OLT

$T_{G}$ : guard time between two successive ONU transmissions

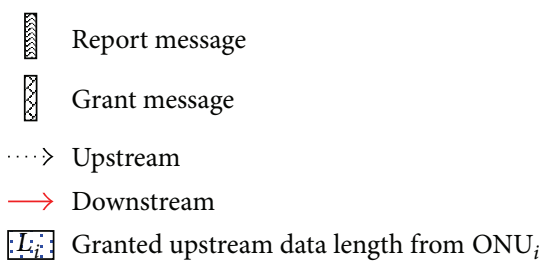

Figure 1: An example for the DP-DBA algorithm under high traffic load conditions.

where the processing time of the DBA algorithm is denoted as $T_{\mathrm{dba}}$, RTT denotes the round-trip time from ONU to OLT, and $T_{\mathrm{ONU}}$ denotes the processing time of the ONU.

The upstream direction of the line is undoubtedly idled because all ONUs are waiting for the grant messages of the next cycle. Such an idle period degrades the performance in terms of bandwidth utilization. To address the idle period problem and excess bandwidth collection, we propose a novel DBA algorithm that combines the Sort-DBA scheme with a dual-polling mechanism for excess bandwidth redistribution. To eliminate the idle time according to the bandwidth demands of all ONUs, the Sort-DBA scheme can be divided into two cases. The two cases are described as follows.

(1) At Least One REPORT Is Long Enough. In Figure 1, during the cycle time $I$, the $R_{N-1}$ is arranged to be the last one to transmit data, and it has enough granted bandwidth length to let the OLT send all the GATEs for the ONUs. Note that we can make the ONU piggyback its REPORT in front of its transmission data by sending the GATE message two times. One GATE message records the transmission time of the REPORT massage. The other records the granted bandwidth and transmission time in the next cycle. To compensate the idle time, the granted bandwidth of the last ONU in cycle time $(I+1)$ should be greater than the $B_{i}^{\mathrm{min}}$ which is expressed as

$$
B_{i}^{\min }=\frac{K_{i} R_{u} \times\left(T_{\text {idle }}-T_{g}\right)}{8},
$$

where $R_{u}$ is the upstream bandwidth, $T_{g}$ is the guard time for the OLT power level adjustment and data processing, and $K_{i}$ varies from the minimum value to the contract value according to the SLA (service level agreement). As for the uniform load pattern, $K_{1}=K_{2}=\cdots=K_{N}=1$ and $B_{i}^{\min }=B_{\min }$. To reduce the delay time, the ONUs transmit their data before the REPORT message except the last ONU. The sequence of transmission ordering is arranged according to the sorting sequence of their granted data size. In Figure 1, the sorted sequence of the granted data size of the ONUs in cycle time $I$ is $L_{N-1} \geq L_{2} \geq L_{N} \geq \cdots \geq L_{5}$. Therefore, $R_{N-1}$ is arranged to be the last one to transmit data. After receiving all the REPORTs from the ONUs, the OLT runs the DBA operation and finds that at cycle time $(I+1)$ there is one ONU (here is the $\mathrm{ONU}_{1}$ ) that matches the requirement of a request length greater than $B_{\min }$. The transmission sequence of other ONUs is assigned according to the sorting order of their requested data size. In this case, the idle time is eliminated completely. If more than one ONU has a request length greater than or equal to $B_{\min }$ in the cycle time, the OLT will select any ONU as the last ONU, completely eliminating the idle time.

(2) No Granted Bandwidths Are Long Enough. The second case is that the OLT cannot find any ONU with REPORT of large enough bandwidth request when all the ONUs are operating at a light traffic load. To deal with this case, a compensation scheme is needed so that other granted data is arranged together during an idle period after the last granted bandwidth so as to use the remainder space of the idle time (designated $\left.L_{\text {ins }}\right)$. One guard time distance $\left(L_{q}\right)$ is necessary between each neighboring granted bandwidth of the ONUs that will be laid out together in the $L_{\text {ins }}$. This is a control overhead of the Sort-DBA scheme. Therefore, to minimize bandwidth waste when using the remainder $L_{\text {ins }}$, each selected granted bandwidth must be larger than $L_{g}$ to avoid extra waste caused by this process. 
2.2. Intrapolling Scheme. During each cycle time, if we can assign the remaining bandwidth of the light load ONUs to the heavy load ONUs, then bandwidth utilization can be improved. Design of the intrapolling mechanism is based on this concept. Two polling tables are needed at OLT. Layer I polling table records the bandwidth request $\left(R_{i}^{r}\right)$ and RTT of each $\mathrm{ONU}_{i}$. Layer II polling table records the extra bandwidth requests $\left(R_{i}^{q}\right)$ from the heavy load ONUs. We can say that the interpolling, which uses Layer I polling table, is used for inter-ONU scheduling. But the intrapolling, which uses Layer II polling table, is used by the OLT to deal with the extra bandwidth distribution. The intrapolling is different from the intra-ONU scheduling, which is performed by the ONU for bandwidth distribution to queues in ONU. Detailed operations are addressed in the following.

(1) A light load $\mathrm{ONU}_{i}$ piggybacks its bandwidth request $\left(R_{i}^{r}\right)$ for the next transmission cycle to the OLT. The OLT grants $\mathrm{ONU}_{i}$ the assigned bandwidth $\left(B_{i}^{g}\right)$ according to its bandwidth request $\left(R_{i}^{r}\right)$. Next the OLT calculates the remaining bandwidth $\left(B_{i}^{r}\right)$ which is defined in the following equation as

$$
B_{i}^{r}=B_{\min }-R_{i}^{r}
$$

The OLT gathers the accumulated remaining bandwidth $\left(B_{\text {excess }}^{\text {total }}\right)$ which is expressed in the following equation as

$$
B_{\text {excess }}^{\text {total }}=\sum_{i=1}^{M} B_{i}^{r}
$$

where $M$ is the number of the light load ONUs. A heavy load $\mathrm{ONU}_{k}$ also piggybacks its bandwidth requirement $\left(R_{k}^{r}\right)$ for the next transmission cycle to the OLT. In addition to $R_{k}^{r}$, an extra request about the extra need $\left(R_{k}^{q}\right)$ for bandwidth is also piggybacked in the transmission data. Again, the OLT grants the $\mathrm{ONU}_{k}$ the assigned bandwidth $\left(B_{k}^{g}\right)$. As for the $R_{k}^{q}$, it is recorded in Layer II polling table. Instead of distributing the remaining bandwidth equal to the heavy load ONUs, we make use of a polling scheme to record their extra bandwidth needs in the intrapolling table. Because of the prohibition of frame fragmentation in IEEE 802.3ah, there is still a possibility that if we share the remaining bandwidth to the heavy load ONUs equally or by their weighting, the bandwidth gap to $B_{\min }$ may waste bandwidth. The granted excess bandwidth $\left(B_{k}^{q}\right)$ for $\mathrm{ONU}_{k}$ is calculated according to the following equation as

$$
B_{k}^{q}= \begin{cases}R_{k}^{q}, & \text { if } R_{k}^{q}<B_{\text {excess }}^{\text {total }} \\ B_{\text {excess }}^{\text {total }}, & \text { if } R_{k}^{q} \geq B_{\text {excess }}^{\text {total }}\end{cases}
$$

If the extra demand of one specific ONU is satisfied, the entry of that ONU at the intrapolling table will be erased. Otherwise, from the fairness point of view, the precedence of the heavy load $\mathrm{ONU}_{k}$ is changed to be the last one in the intrapolling table if it is served in this round of excess bandwidth allocation. Also, $R_{k}^{q}$ is updated as $\left(R_{k}^{q}-B_{k}^{q}\right)$. Values in Layer I and Layer II polling tables are overwritten with the latest values from reports from ONUs. Except for the
TABLE 1: Polling tables of the DP-DBA algorithm.

(a) Layer I polling table

\begin{tabular}{lcc}
\hline ONU & $R_{i}^{r}$ & RTT \\
\hline 1 & 12,000 & 600 \\
2 & 23,000 & 880 \\
3 & 24,375 & 750 \\
4 & 7,900 & 1,100 \\
5 & 23,900 & 1,000 \\
\hline
\end{tabular}

(b) Layer II polling table

\begin{tabular}{lc}
\hline ONU & $R_{i}^{q}$ \\
\hline 2 & 20,000 \\
3 & 50,000 \\
5 & 19,000 \\
\hline
\end{tabular}

(c) Layer II polling table after intrapolling

\begin{tabular}{lc}
\hline ONU & $R_{i}^{q}$ \\
\hline 5 & 19,000 \\
3 & 39,300 \\
\hline
\end{tabular}

information of the served ONUs in Layer II, values of the fields in both tables are overwritten.

We illustrate the DP-DBA operation with the following example. The network settings are shown in Figure 2. Suppose that there are five ONUs in the system, $\mathrm{ONU}_{1}, \mathrm{ONU}_{2}, \ldots, \mathrm{ONU}_{5}$. The distance between the ONUs and OLT is set as $20 \mathrm{Km}$. The SLA is the same for the ONUs. Other parameters are $T_{g}=1 \mathrm{us}, R_{u}=1 \mathrm{~Gb} / \mathrm{s}, T_{\mathrm{DBA}}=0$, and $T_{\text {ONU }}=0$. Based on (1), we calculate $B_{\min }=24.375$ Kbytes. At cycle time $I$, after all the requests arrive at the OLT, $R_{i}^{r}$ is recorded as in Table 1 (a) and $R_{i}^{q}$ is recorded as in Table 1(b). Here, the bandwidth requests are set as follows: $R_{1}^{r}=12 \mathrm{~KB}, R_{2}^{r}=23 \mathrm{~KB}, R_{3}^{r}=24.375 \mathrm{~KB}, R_{4}^{r}=7.9 \mathrm{~KB}$, and $R_{5}^{r}=23.9 \mathrm{~KB}$. The extra need in bandwidth is set as follows: $R_{2}^{q}=20 \mathrm{~KB}, R_{3}^{q}=50 \mathrm{~KB}$, and $R_{5}^{q}=19 \mathrm{~KB}$. Consider the remaining bandwidth in (3) and the prohibition of frame fragmentation. The remaining bandwidths $\left(B_{i}^{r}\right)$ of each ONU are $B_{1}^{r}=12.375 \mathrm{~KB}, B_{2}^{r}=1.375 \mathrm{~KB}, B_{4}^{r}=16.475 \mathrm{~KB}$, and $B_{5}^{r}=0.475 \mathrm{~KB}$. Then, we have $B_{\text {excess }}^{\text {total }}=30.7 \mathrm{~KB}$ which will be distributed to $\mathrm{ONU}_{2}$ and then $\mathrm{ONU}_{3}$ in order. $\mathrm{ONU}_{3}$ 's extra bandwidth need is partially satisfied and set to be the last one with lowest priority of the intrapolling table. Note the gap between the requested bandwidth and $B_{\min }$ of $\mathrm{ONU}_{2}$ in Table 1 , which is a consequence of packet fragmentation being prohibited in IEEE 802.3ah.

OLT checks the intrapolling table and decides to assign $B_{2}^{q}=20 \mathrm{~KB}$ (first priority in the table) and $B_{3}^{q}=10.7 \mathrm{~KB}$ (second priority). But $\mathrm{ONU}_{3}$ still has extra need for bandwidth. For a fairness basis, the precedence of $\mathrm{ONU}_{3}$ is now changed to be the least one. The updated intrapolling table is shown in Table 1(c).

Considering the packet header and the guard time overhead, we can calculate the average system throughput as 


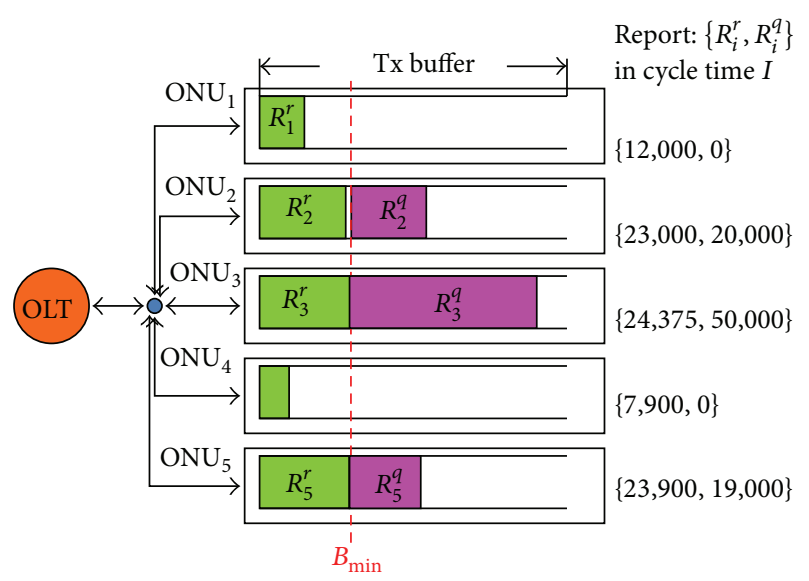

FIGURE 2: ONU bandwidth requests in the intrapolling scheme for cycle time $I$.

follows. Here $H$ is the size of the packet header and $N$ is the number of ONUs. The number of light load ONUs is $M$ :

$$
\eta_{\mathrm{av}}=\frac{\sum_{i=1}^{N} B_{i}^{r}+\sum_{i=1}^{M} B_{i}^{q}}{N\left(H+T_{g} R_{u}\right)+\sum_{i=1}^{N} B_{i}^{r}+\sum_{i=1}^{M} B_{i}^{q}} .
$$

The maximum frame length of the system can be expressed as

$$
T_{\max }=N\left(H+T_{g} R_{u}\right)+\sum_{i=1}^{N} B_{i}^{r}+\sum_{i=1}^{M} B_{i}^{q} .
$$

\section{Analytical Delay}

In this section, we propose two models for the IPACT-limited and Sort-DBA schemes' delay analysis. With the analytical equations in Section 3.2, we can explain why the Sort-DBA scheme outperforms the IPACT-limited scheme when the network is medium-loaded. From the model described in Section 3.1, we can see why the improvement made by the Sort-DBA scheme decreases as the network is heavy-loaded.

In Section 3.1, from the viewpoint of a specific frame arriving at the ONU buffer, we derive a general analytical equation to estimate the delay experienced by this specific frame. Next, in Section 3.2, from the viewpoint of unbalanced ONU loads, we derive equations for deferred traffic of both schemes. The deferred traffic has to wait for another cycle time or more to be delivered. Therefore, less deferred traffic means less average packet delay.

3.1. Estimated Delay from the Point of a Specific Frame. Here, we assume there is a specific frame, $\alpha$, which arrives at the ONU buffer and sees that there are already $S_{\text {LOG }}$ bytes in buffer before him. Intuitively, the frames ahead of $\alpha$ must be transmitted and then $\alpha$ can be delivered. In this part, we assume all the ONU loads are balanced. We vary the maximum cycle time $T_{\max }$ and $S_{\mathrm{LOG}}$ to see the delay experienced by $\alpha$. Other system parameters are listed as follows: guard time: (= $1 \mathrm{us}$ ), idle time: $T_{\text {idle }}(=0 \mathrm{us})$, number

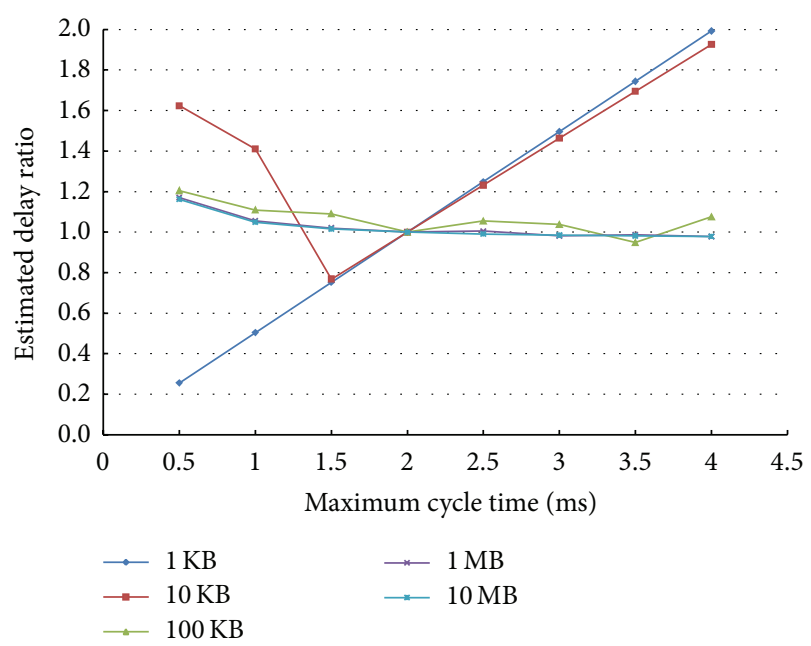

FIgURE 3: Estimated delay ratio.

of ONUs: $N(=16)$, line rate (upstream): $R_{U}$ (=1 Gbps), report size: $L_{\text {request }}\left(=64\right.$ bytes), and unused slot remainder: $L_{\text {USR }}(=$ 503 bytes). First, we calculate the effective granted bandwidth of each ONU during a cycle time:

$$
\begin{aligned}
\mathrm{BW}_{\text {effective }}= & \frac{R_{U}}{N} \times\left(T_{\max }-N \times T_{g}-T_{\text {idle }}\right) \\
& -L_{\text {request }}-L_{\text {USR }} .
\end{aligned}
$$

The frames before $\alpha$ may take several rounds of cycles to be delivered. $[x]$ is a function to take the round-down value of $x$. Besides, according to the polling mechanism, $\alpha$ averagely has to wait half of the cycle time to be reported to the OLT. Now, we derive the average delay equation:

$$
\begin{aligned}
D= & {\left[\frac{S_{\text {LOG }}}{\mathrm{BW}_{\text {effective }}}\right] \times T_{\max }+\frac{1}{R_{U}} } \\
& \times\left(S_{\text {LOG }}-\left[\frac{S_{\text {LOG }}}{\mathrm{BW}_{\text {effective }}}\right] \times \mathrm{BW}_{\text {effective }}\right)+\frac{T_{\text {max }}}{2} .
\end{aligned}
$$

We vary the maximum cycle time from $0.5 \mathrm{~ms}$ to $4 \mathrm{~ms}$. With $S_{\text {LOG }}$ ranging from $1 \mathrm{~KB}$ to $10 \mathrm{MB}$, Figure 3 shows the delay ratio value with different parameter settings. The $2 \mathrm{~ms}$ (the maximum cycle time) case is used as the normalized basis.

From the aspect of $S_{\mathrm{LOF}}$, taking the $1 \mathrm{~KB}$ (blue line with diamond shape) case, for example, we can tell that larger $T_{\max }$ incurs more delay. However, as for the $1 \mathrm{MB}$ case, the increase of $T_{\max }$ can slightly decrease the delay. This accounts for the phenomenon that as the network is heavy-loaded, the slight improvement is made by the Sort-DBA scheme. However, it also implies that increasing $T_{\max }$ is not the main reason which accounts for the delay improvement made by the Sort-DBA scheme when the network is medium-loaded. With careful examination, we find that the answer lies in the unbalanced ONU loads.

3.2. Delay Analysis under Unbalanced ONU Loads. With considering the unbalanced ONU loads, we analyze the delay 
performance of the IPACT-limited and Sort-DBA schemes. Rather than calculation of the delay, we calculate the amount of the deferred traffic during a cycle round for both schemes. It is easy to figure out that if there is more deferred traffic in the IPACT-limited scheme than in the Sort-DBA scheme, more time will be taken for the IPACT-limited scheme to deliver the deferred traffic. Accordingly, the delay performance of the IPACT-limited scheme is deteriorated.

Here are the system parameters: number of ONUs: $N$ (= 16), maximum transmission window size: $\mathrm{BW}_{\text {IPACT-limited }}^{\mathrm{min}}(=$ $15 \mathrm{~KB}), \mathrm{BW}_{\text {Sort-DBA }}^{\min }(=24.875 \mathrm{~KB})$, total request bandwidth $\mathrm{BW}_{\text {total }}^{\text {request }}(=180 \mathrm{~K}$ to $240 \mathrm{~K})$, number of unbalanced $\mathrm{ONU}$ pairs ( $=1$ to 7$)$, and unbalanced factor $\beta(=0.5$ to 0.8$)$.

We use $K$ and $\beta$ to mimic different conditions of the unbalanced ONU loads. For simplicity, we assume there are only three kinds of ONUs, namely, heavy-loaded ONU, medium-loaded ONU, and light-loaded ONU. The traffic load ratio of them is $(1+\beta): \beta:(1-\beta)$. Now, under the same $\mathrm{BW}_{\text {total }}^{\text {request }}$, we derive the total granted bandwidth equations for both schemes:

$$
\begin{aligned}
\mathrm{BW}_{\text {total-grant }}^{\mathrm{IPACT} \text {-limited }}= & K \times\left\{\min \left[(1+\beta) \times \frac{\mathrm{BW}_{\text {total }}^{\text {grant }}}{N}, 15 K\right]\right. \\
& \left.+(1+\beta) \times \frac{\mathrm{BW}_{\text {total }}^{\text {grant }}}{N}\right\} \\
& +(N-2 K) \times \frac{\mathrm{BW}_{\text {total }}^{\text {grant }}}{N}, \\
\mathrm{BW}_{\text {total-grant }}^{\text {Sort-DBA }}= & K \times\left\{\min \left[(1+\beta) \times \frac{\mathrm{BW}_{\text {total }}^{\text {grant }}}{N}, 24.875 K\right]\right. \\
& \left.+(1+\beta) \times \frac{\mathrm{BW}_{\text {total }}^{\text {grant }}}{N}\right\} \\
& +(N-2 K) \times \frac{\mathrm{BW}_{\text {total }}^{\text {grant }} .}{N} .
\end{aligned}
$$

With the parameter settings, $\mathrm{BW}_{\text {total }}^{\text {request }}=\{180 K, 200 K, 220 K$, $240 K\}$ and $\beta=\{0.5,0.8\}$, Figure 4 shows the ratio of $\mathrm{BW}_{\text {total-grant }}^{\text {IPACT-limited }}$ to $\mathrm{BW}_{\text {total-grant }}^{\text {Sort-DBA }}$ as a function of number of unbalanced ONU pairs.

With the plot, we can tell that with the same $\mathrm{BW}_{\text {total }}^{\text {request }}$ increasing of unbalanced ONU pairs results in more reduction of the ratio value. This means there is more deferred traffic in the IPACT-limited scheme. Accordingly, it brings delay improvement to the Sort-DBA scheme over the IPACTlimited scheme. So, we may draw the conclusion that the degree of unbalanced loads has a significant influence on the delay performance of DBA schemes. In addition, the increase of $\beta$ also results in the reduction of the ratio value which implies improvement in delay performance of the Sort-DBA scheme over the IPACT-limited scheme. Now, we can explain the delay improvement of the Sort-DBA scheme when the network is medium-loaded. But why does the improvement

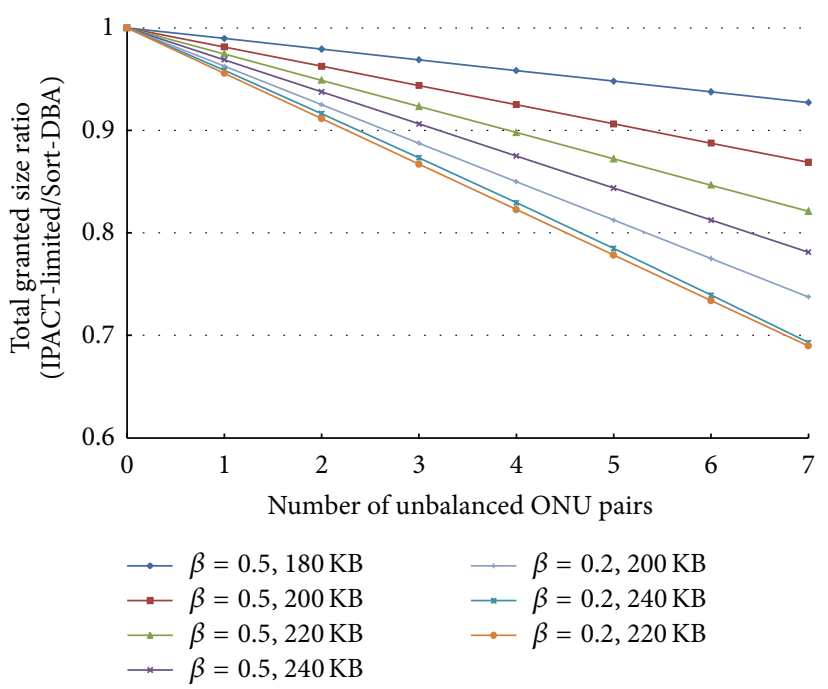

FIGURE 4: Total granted size ratio.

decrease as the network becomes heavy-loaded? The answer lies in the loss of the unbalance. When the requests of all ONUs grow to the maximum transmission window size, there is nearly no unbalanced ONU load and the difference of deferred traffic between the two schemes decreases. The situation now goes back to the model mentioned in the previous section and the improvement becomes less significant.

\section{Simulation Results and Discussions}

We compare the performance of our proposed DP-DBA to five other well-known schemes, that is, IPACT with fixed service (IPACT-fixed), IPACT with limited service (IPACTlimited), DBA2 with fair-excess (DBA2-FE), Double-Phase Polling (DPP), and Sort-DBA $[11,12,16,17]$. The performance metrics include average transfer delay and average grant length. Simulations are conducted by SIMSCRIPT II.5. The simulation setup parameters are described as follows. We use the Poisson traffic generation for packet arrival. The interarrival time of the packets follows the exponential distribution. The IP packet length at each ONU is generated according to the packet size distribution of the MCI backbone OC-3 links [22]. The mean size is about 353.8 bytes. The upstream total bandwidth $\left(R_{u}\right)$ is 1 Gbps. The distance between the OLT and each ONU is $20 \mathrm{Km}$. The buffer size at an ONU is 1 Mbyte. The guard time $\left(R_{u}\right)$ is $1 \mu \mathrm{s}$. The size of the REPORT is 64 bytes. The number of ONUs is 15 . For the IPACT-fixed, IPACT-limit, and DBA2-FE schemes, $B_{\min }$ is calculated to be 15 Kbytes according to their definition. For the Sort-DBA and DP-DBA schemes, $B_{\text {min }}$ is defined as 24.876 Kbytes. In addition to using the uniform load of the ONUs as input, we also make use of the asymmetrical load to evaluate the performance in terms of the transfer delay, average queue delay, and average granted data length.

4.1. ONU Balanced Traffic Load. Figure 5 shows the simulation results for the average transfer delay of five schemes, 


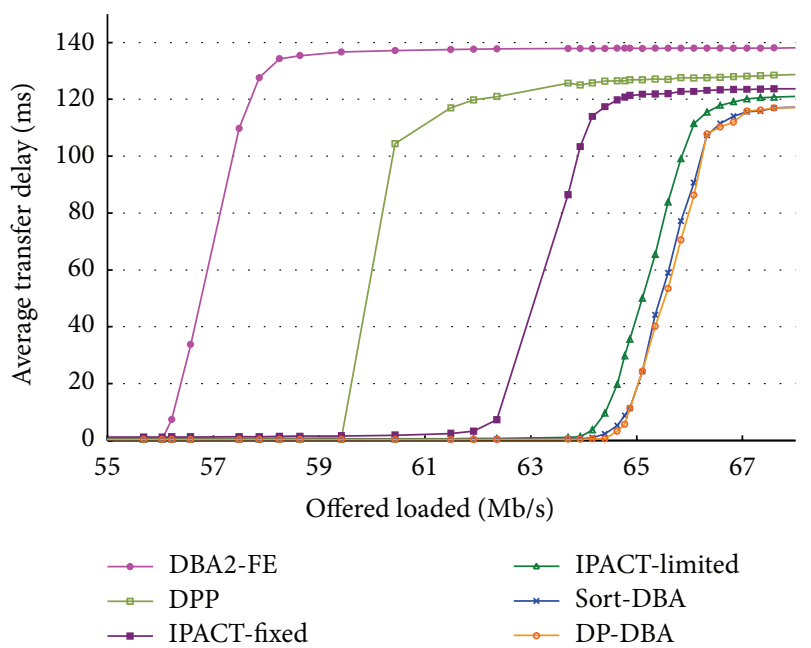

FIGURE 5: Average transfer delay per ONU for IPACT-fixed, IPACTlimited, DPP, DBA2-FE, Sort-DBA, and DP-DBA schemes under ONU balanced traffic load.

that is, IPACT-fixed, IPACT-limited, DBA2-FE, Sort-DBA, and our DP-DBA. Here an ONU uniform traffic load pattern is used. Later, the simulation results will illustrate how the ONU traffic load pattern affects the performance of all five schemes. As seen in Figure 5, the $x$-axis is the offered load and the $y$-axis is the average transfer delay. At about $56 \mathrm{Mbps}$, the average transfer delay of the DBA2-FE scheme rises quickly. For the other schemes, the curves rise at approximately 62 64 Mbps. We can see that the DP-DBA and Sort-DBA perform the best among the different schemes. This is because the REPORT message of the last ONU is delivered in advance of the data transfer, so DBA operation at the OLT can be started early. Under the condition of at least one heavy load ONU, the idle time problem can be solved completely since the OLT can deliver GATE messages to all ONUs in time. This significantly reduces waiting time and wasted bandwidth. In contrast, if all ONUs have heavy load requests above $B_{\min }$, the early allocation mechanism of DBA2 with a fair-excess scheme cannot solve the idle time problem perfectly, resulting in decreased system performance. Regarding the IPACT-fixed scheme, the fixed bandwidth assignment mechanism cannot deal with the idle time issue so it performs better than the DBA2-FE scheme. However, the assigned bandwidth of the light load ONUs is wasted, thus lowering the performance. In the DPP scheme, we can see the delay improvement of the DPP scheme over the DBA2-FE scheme. With this reason, due to the group separation, there is the possibility that the idle time appears when the loads of the other group are not large enough. The performance of the DPP scheme lies between the DBA2-FE and the IPACT-limited schemes.

Figure 6 shows the average granted data length as a function of the offered load under an ONU balanced traffic load. At medium load, the Sort-DBA and DP-DBA schemes increase the demand length of each ONU to $B_{\min }$ to eliminate idle time, so the average granted data length per ONU is more than the other DBA schemes. However, DP-DBA has a shorter granted data length than the Sort-DBA scheme at

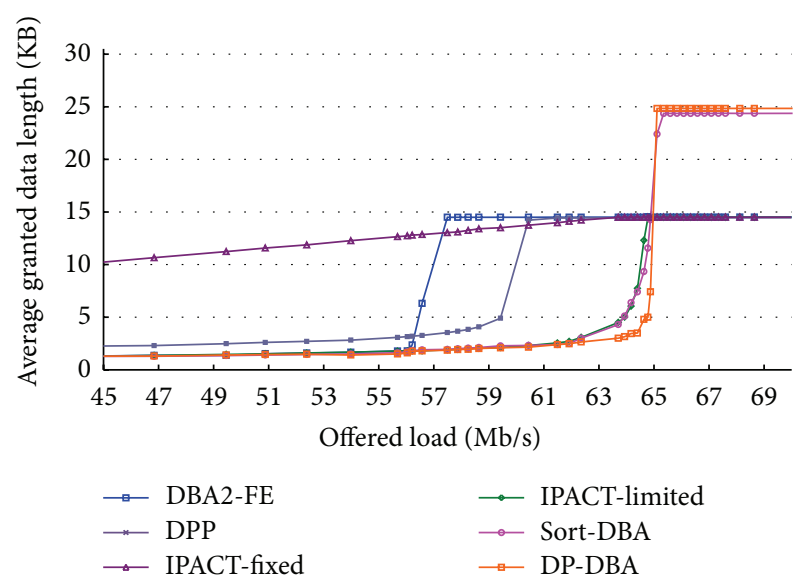

Figure 6: Average granted data length per ONU for IPACT-fixed, IPACT-limited, DPP, DBA2-FE, Sort-DBA, and DP-DBA schemes.

heavy load. The reason is that the intrapolling scheme of DPDBA makes use of remaining bandwidth of the overloaded ONUs to reduce the average granted data length. For IPACTfixed and IPACT-limited schemes, a maximum transmission window of 15 Kbytes is specified for each ONU to ensure that the cycle time is no more than $2 \mathrm{~ms}$ for delay-sensitive applications. As for the IPACT-fixed scheme with our traffic model, we find that the average granted data length per ONU in one cycle under heavy load is only $14.5 \mathrm{Kbytes}$. This is because the prohibition of fragmentation to Ethernet frames makes the ONU unable to fully utilize the maximum transmission window with FIFO queue discipline. The average granted data length with the IPACT-limited scheme is even smaller than that with IPACT-fixed scheme for light to high loads because the IPACT-limited scheme always grants the requested data with the real load demand of each ONU so as to obtain the shortest transmission cycle. The average granted data length with the DBA2-FE scheme is close to 15 Kbytes. This is because the DBA2-FE scheme always allocates the remaining idle slots to other ONUs when the transmission demand is excessive. In this figure, we can also find that the average granted data length of the DPP scheme increases rapidly when the offered load goes beyond $59 \mathrm{Mbps}$. This is consistent with the delay performance of the DPP scheme in Figure 5.

The performance difference between DP-DBA and the other schemes is not significant because under ONU uniform traffic load conditions all ONUs are under $B_{\text {min }}$ or all ONUs need extra bandwidth to transmit data. Therefore, the intrapolling scheme of DP-DBA cannot be used efficiently since, under heavy load condition, the remaining bandwidth for reallocation is very limited.

4.2. ONU Asymmetrical Traffic Load. In real EPON systems, the ONU traffic load pattern is not uniform. Thus, we simulate the performance of the same five schemes mentioned in Section 4.1 in terms of average transfer delay, average queuing delay, and average granted data length. In fact, it will be seen that our proposed DP-DBA performs well, especially in 


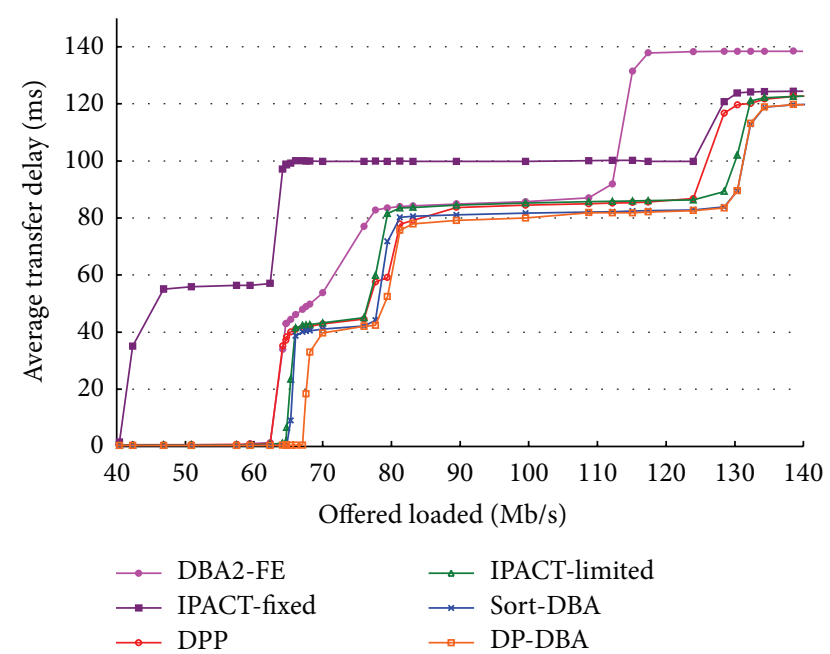

FIgURE 7: Average transfer delay per ONU for the IPACT-fixed, IPACT-limited, DPP, DBA2-FE, Sort-DBA, and DP-DBA schemes under ONU asymmetrical traffic load.

the asymmetrical load case for our intrapolling scheme. The asymmetrical settings are described as follows. The number of ONUs is 15 and one-third of them are light load, onethird are medium load, and one third are heavy load. The bandwidth requirement ratio of light load, medium load, and heavy load is $1: 2: 3$. Other settings are the same as those used in part Section 4.1. Referring to Figure 4, the simulation setting is close to the number of unbalanced ONU pairs being equal to 5 and the unbalanced factor $\beta$ being equal to 0.5 . In the following figures, we will see that the ratio of the granted bandwidth size in IPACT-LS over that in the IPACTlimited scheme over that in DP-DBA decreases more when applying the asymmetric traffic load as compared to that with symmetric traffic load.

The average ONU transfer delay is shown in Figure 7. The $x$-axis is the offered load (Mbps) which varies according to the generated traffic at each ONU. The $y$-axis is the simulation results for the average transfer delay measured in milliseconds. We can see the difference of average transfer delay for the balanced traffic load (Figure 5) and the asymmetrical traffic load. There are three sections of strongly rising curves among the five schemes. The initial rising sections are due to the heavy load ONUs. Figure 7 shows that DP-DBA performs the best because it is the last curve to start rising, that is, at about $67 \mathrm{Mbps}$. Excepting the IPACT-fixed scheme which starts rising early, DP-DBA can make use of $2 \mathrm{Mbps}$ more than the Sort-DBA and IPACT-limited schemes. This is because of the intrapolling scheme of DP-DBA. The IPACTfixed scheme uses the fixed bandwidth allocation mechanism and wastes most of the bandwidth which can be reassigned to the heavy load ONUs, which leads to early rising in the average transfer delay. As the traffic load increases, the medium load ONUs start to contribute to rising average transfer delay. Finally, all the ONUs are overloaded, and there is little performance difference among the four schemes except the DBA2-FE. This is because there is little room

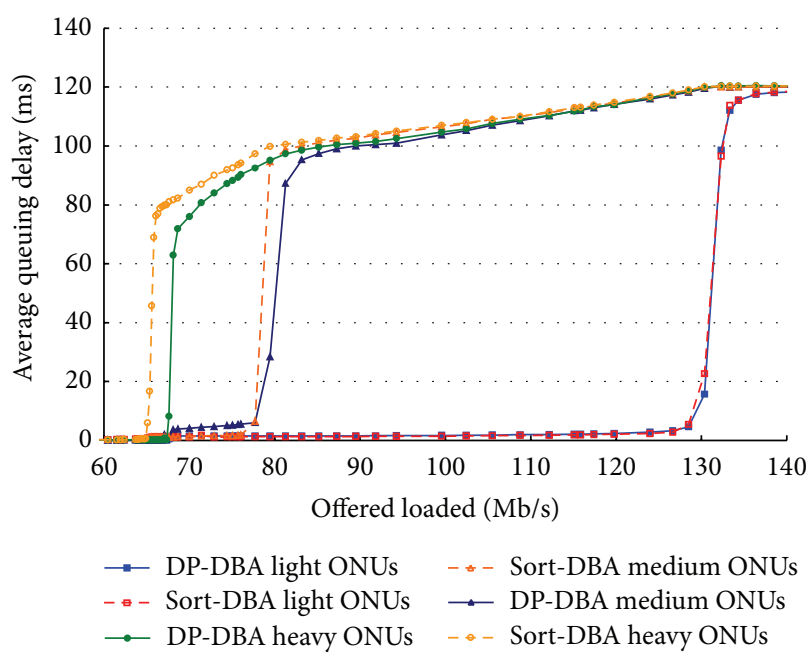

FIGURE 8: ONU average queuing delay under an asymmetrical load pattern.

for bandwidth to be assigned for heavy load ONUs. Thus, DP-DBA performs as well as the other schemes. Note that the DBA2-FE scheme suffers from more average transfer delay than the other schemes under heavy load conditions. This is due to the failure of the early allocation mechanism, which results from all ONUs being overloaded. The idle time problem results in significant increase to the average transfer delay of the DBA2-FE scheme.

In Figure 7, the average transfer delay of the DPP scheme behaves similarly to other schemes under ONU asymmetrical traffic load. When the offered load goes beyond $120 \mathrm{Mb} / \mathrm{s}$ and that means all ONUs are heavy-loaded, the performance of the DPP scheme is close to the IPACT-limited scheme.

We collect the average queuing delay results of DP-DBA and the Sort-DBA scheme for further analysis regarding the performance difference between them under varying offered traffic load conditions. Figure 8 shows that, under light offered traffic load, the heavy load ONUs of DP-DBA can achieve $67 \mathrm{Mbps}$ while those ONUs of the Sort-DBA scheme achieve only $65 \mathrm{Mbps}$. This is because the intrapolling mechanism of DP-DBA helps the heavy load ONUs make use of the unused bandwidth of the medium and light load ONUs. As the offered load increases, there is less bandwidth that can be allotted to ONUs with extra bandwidth requirements. Then the performance difference between DP-DBA and the Sort-DBA schemes becomes less significant.

Figure 9 shows the average granted data length at the ONUs for the five schemes. According to the simulation settings, $B_{\min }$ is calculated as $15 \mathrm{Kbytes}$ for the IPACT-fixed, IPACT-limited, and DBA2-FE schemes and 24.876 Kbytes for the Sort-DBA and DP-DBA schemes. DP-DBA still performs the best among the five schemes because the average granted data length of ONUs can fully utilize the bandwidth $B_{\min }$ with the help of intrapolling table. The Sort-DBA and IPACT-limited schemes also have the ability to adjust the frame size dynamically according to the bandwidth requests from the ONUs. However, they do not allot the remaining 


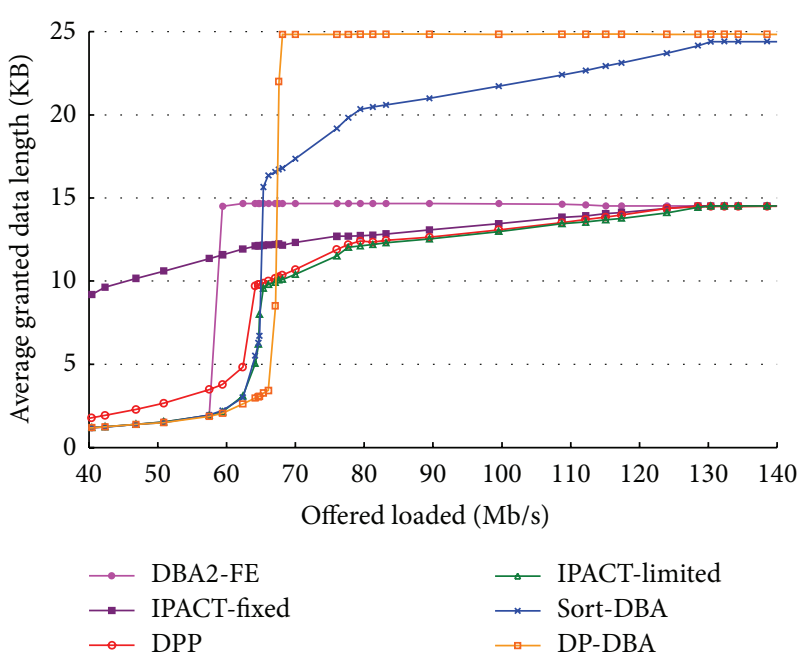

FIGURE 9: Average granted data length per ONU for IPACT-fixed, IPACT-limited, DBA2-FE, DP-DBA, and Sort-DBA schemes under an asymmetrical traffic load.

bandwidth to the heavy load ONUs. According to the IEEE 802.3ah, frame fragmentation is prohibited, resulting in the frame size of the IPACT-limited scheme being 14.5 Kbytes instead of $15 \mathrm{Kbytes}\left(B_{\min }\right)$. The DBA2-FE scheme achieves the least average granted data length due to the idle time problem which comes from failure of the early allocation scheme. Meanwhile, the frame fragmentation problem of IEEE 802.3ah also contributes to the decrease of the average granted data length. Note that, under an asymmetrical traffic load, the DBA2-FE scheme cannot perform as well as it does in the balanced load condition. Besides, the average granted data length the DPP scheme grows in a way similar to that of the IPACT-limited scheme, especially when the network load is larger than $70 \mathrm{Mb} / \mathrm{s}$.

The presented simulation results show that the proposed DP-DBA method achieves higher utilization under most load conditions than the other DBA algorithms. With the help of the intrapolling mechanism, the remaining bandwidth can be assigned to the heavy load ONUs, thereby reducing the queuing time of the data. The idle time can be eliminated if there is at least one ONU which requests bandwidth more than $B_{\min }$. DP-DBA performs better when the ONU traffic load pattern is asymmetrical. Such settings are closer to the real world network environment.

\section{Conclusion}

This paper has proposed a dual-polling DBA algorithm for EPON systems. The proposed scheme consists of two main elements, namely, the interpolling and intrapolling mechanisms. Interpolling mechanism is designed to deal with the idle time problem. For choosing one $\mathrm{ONU}_{i}$ whose request bandwidth is larger than $B_{\min }$, the OLT assigns the $\mathrm{ONU}_{i}$ to be the last of the transmission ONU sequence and makes $\mathrm{ONU}_{i}$ transmit its REQUEST before data transmission. The transmission order of the other ONUs is arranged according to their bandwidth REQEST size. After collecting the unused bandwidth from the light load ONUs, the intrapolling mechanism helps in assigning the unused bandwidth from the light load ONUs to the heavy load ONUs. It works very well when the ONU load pattern is asymmetrical. The proposed DP-DBA is being compared with the IPACT, DBA2-FE, and Sort-DBA schemes in terms of average transfer delay and average granted data length. Simulation results show that DPDBA outperforms the other schemes when the offered traffic load is experiencing overload conditions. In particular when the traffic pattern is asymmetrical, DP-DBA is superior to the other systems because of the intrapolling mechanism. By reallocation of the unused bandwidth from the light load ONUs to the heavy load ONUs, the average transfer delay can be reduced significantly thanks to the reduction of packet queuing time at the ONUs.

\section{Conflict of Interests}

The authors declare that there is no conflict of interests regarding the publication of this paper.

\section{Acknowledgment}

The authors would like to thank the Ministry of Science and Technology (MOST) of Taiwan for supporting this research under Project no. MOST 103-2622-E-151-006-CC3.

\section{References}

[1] L. Hutcheson, "FTTx: current status and the future," IEEE Communications Magazine, vol. 46, no. 7, pp. 90-95, 2008.

[2] H. Shinohara, "Broadband access in Japan: rapidly growing FTTH market," IEEE Communications Magazine, vol. 43, no. 9, pp. 72-78, 2005.

[3] P. B. Harboe and J. R. Souza, "Passive optical network: characteristics, deployment, and perspectives," IEEE Latin America Transactions, vol. 11, no. 4, pp. 995-1000, 2013.

[4] D. Law, D. Dove, J. D’Ambrosia, M. Hajduczenia, M. Laubach, and S. Carlson, "Evolution of ethernet standards in the IEEE 802.3 working group," IEEE Communications Magazine, vol. 51, no. 8, pp. 88-96, 2013.

[5] M. P. McGarry and M. Reisslein, "Investigation of the DBA algorithm design space for EPONs," Journal of Lightwave Technology, vol. 30, no. 14, pp. 2271-2280, 2012.

[6] A. Mercian, M. P. McGarry, and M. Reisslein, "Impact of report message scheduling (RMS) in 1G/10G EPON and GPON," Optical Switching and Networking, vol. 12, pp. 1-13, 2014.

[7] M. P. McGarry, M. Reisslein, F. Aurzada, and M. Scheutzow, "Shortest propagation delay (SPD) first scheduling for EPONs with heterogeneous propagation delays," IEEE Journal on Selected Areas in Communications, vol. 28, no. 6, pp. 849$862,2010$.

[8] J. Ahmed, J. Chen, L. Wosinska, B. Chen, and B. Mukherjee, "Efficient inter-thread scheduling scheme for long-reach passive optical networks," IEEE Communications Magazine, vol. 51, no. 2, pp. S35-S43, 2013.

[9] Q. Cui, T. Ye, T. T. Lee, W. Guo, and W. Hu, "Stability and delay analysis of EPON registration protocol," IEEE Transactions on Communications, vol. 62, no. 7, pp. 2478-2493, 2014. 
[10] R. Kaler, P. Teotia, and R. S. Kaler, "Simulation of FTTH at 10 Gbit/s for 8 OTU by GE-PON architecture," Optik, vol. 122, no. 22, pp. 1985-1989, 2011.

[11] G. Kramer, B. Mukherjee, and G. Pesavento, "IPACT: a dynamic protocol for an Ethernet PON (EPON)," IEEE Communications Magazine, vol. 40, no. 2, pp. 74-80, 2002.

[12] C. M. Assi, Y. Ye, S. Dixit, and M. A. Ali, "Dynamic bandwidth allocation for quality of service over Ethernet PONs," IEEE Journal on Selected Areas in Communications, vol. 21, no. 9, pp. 1467-1477, 2003.

[13] A. R. Dhaini, C. M. Assi, M. Maier, and A. Shami, "Dynamic wavelength and bandwidth allocation in hybrid TDM/WDM EPON networks," Journal of Lightwave Technology, vol. 25, no. 1, pp. 277-286, 2007.

[14] I. S. Hwang, Z. D. Shyu, L. Y. Ke, and C. C. Chang, "A novel early DBA mechanism with prediction-based fair excessive bandwidth allocation scheme in EPON," Computer Communications, vol. 31, no. 9, pp. 1814-1823, 2008.

[15] J. Zheng, "Efficient bandwidth allocation algorithm for ethernet passive optical networks," IEE Proceedings: Communications, vol. 153, no. 3, pp. 464-468, 2006.

[16] S. Y. Choi, S. Lee, T.-J. Lee, M. Y. Chung, and H. Choo, "Double-phase polling algorithm based on partitioned ONU subgroups for high utilization in EPONs," Journal of Optical Communications and Networking, vol. 1, no. 5, Article ID 5259914, pp. 484-497, 2009.

[17] W.-P. Chen, W.-F. Wang, and W.-S. Hwang, "Adaptive dynamic bandwidth allocation algorithm with sorting report messages for Ethernet passive optical network," IET Communications, vol. 4, no. 18, pp. 2230-2239, 2010.

[18] C. A. Chan, M. Attygalle, and A. Nirmalathas, "Local-trafficredirection-based dynamic bandwidth assignment scheme for EPON with active forwarding remote repeater node," IEEE/OSA Journal of Optical Communications and Networking, vol. 3, no. 3, pp. 245-253, 2011.

[19] D. Nikolova, B. van Houdt, and C. Blondia, "Dynamic bandwidth allocation algorithms for ethernet passive optical networks with threshold reporting," Telecommunication Systems, vol. 28, no. 1, pp. 31-52, 2005.

[20] C.-C. Sue, K.-C. Chuang, Y.-T. Wu, S.-J. Lin, and C.-C. Liu, "Active intra-ONU scheduling with proportional guaranteed bandwidth in long-reach EPONs," Photonic Network Communications, vol. 27, no. 3, pp. 106-118, 2014.

[21] B. Chen, J. Chen, and S. He, "Efficient and fine scheduling algorithm for bandwidth allocation in ethernet passive optical networks," IEEE Journal on Selected Topics in Quantum Electronics, vol. 12, no. 4, pp. 653-659, 2006.

[22] I. White, M. Rogge, K. Shrikhande, and L. Kazovsky, "Design of a control-channel-based media-access-control protocol for HORNET," Journal of Optical Networking, vol. 1, no. 12, pp. 460473, 2002. 


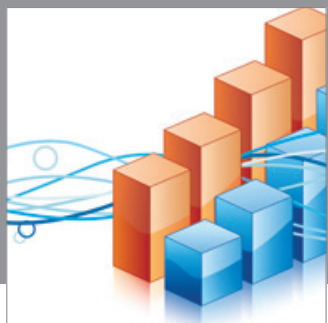

Advances in

Operations Research

mansans

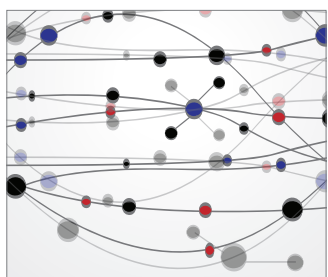

The Scientific World Journal
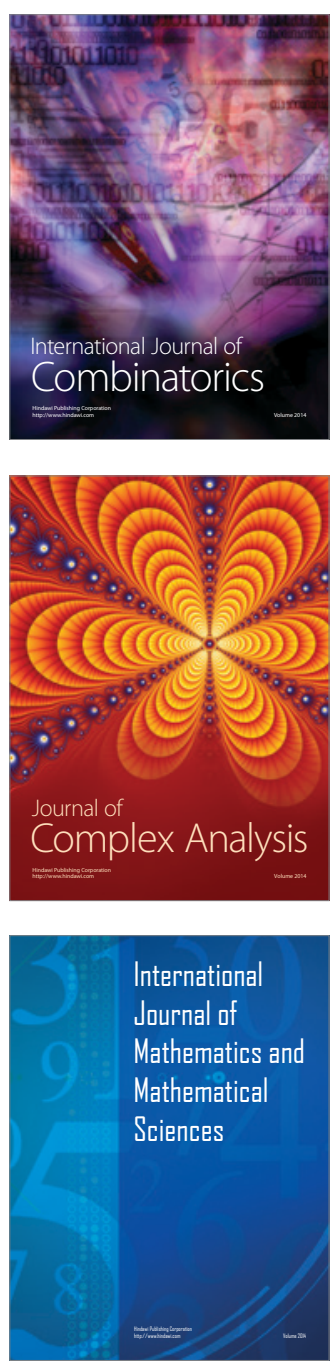
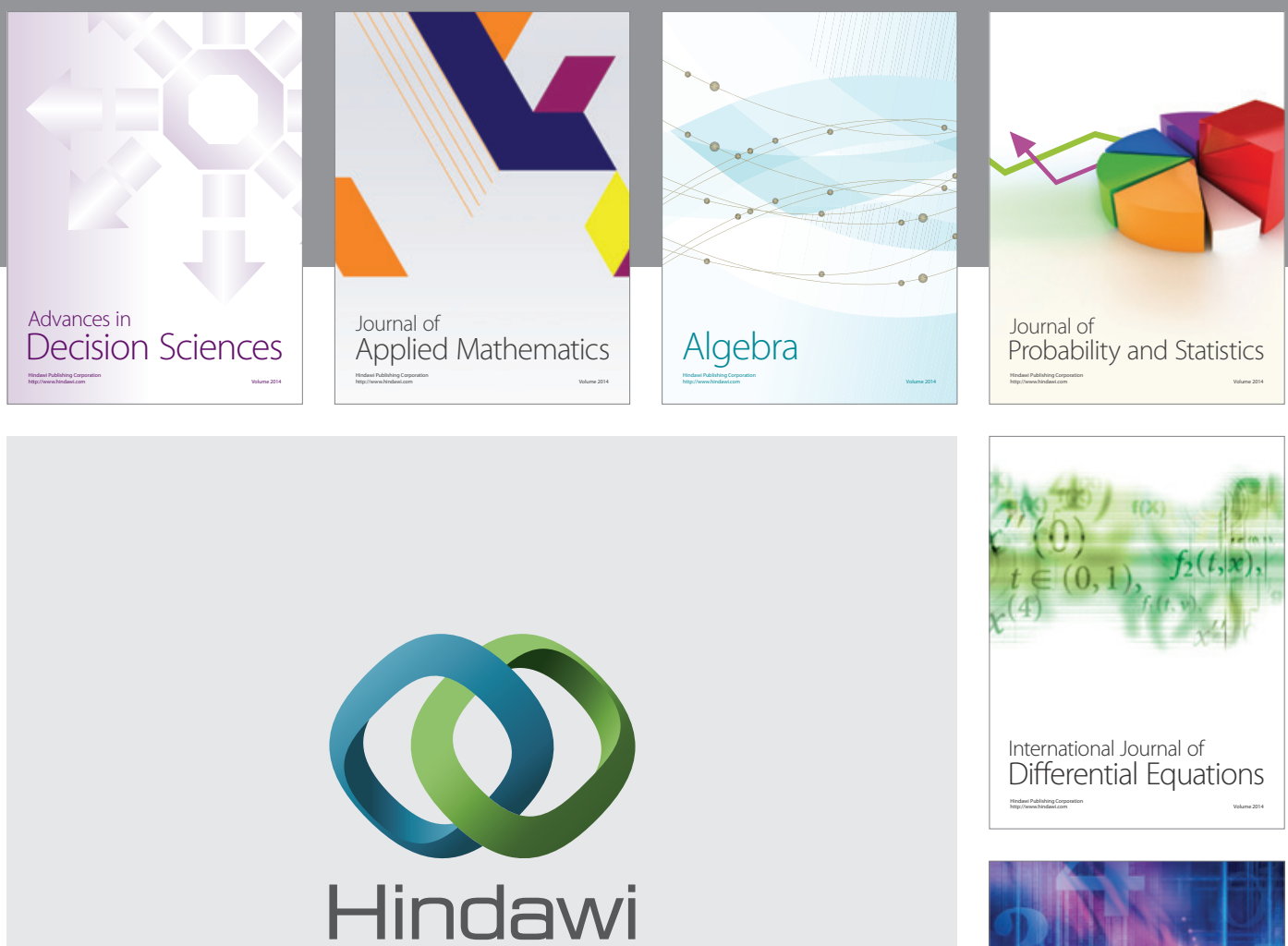

Submit your manuscripts at http://www.hindawi.com
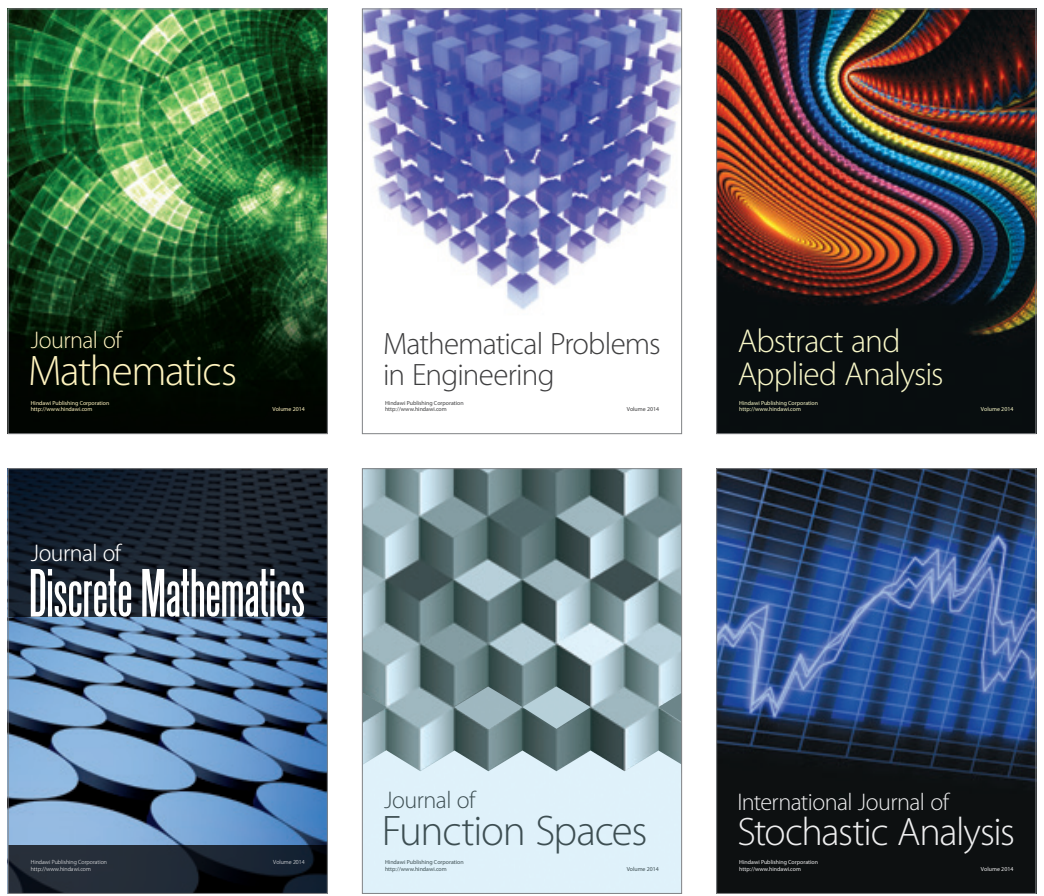

Journal of

Function Spaces

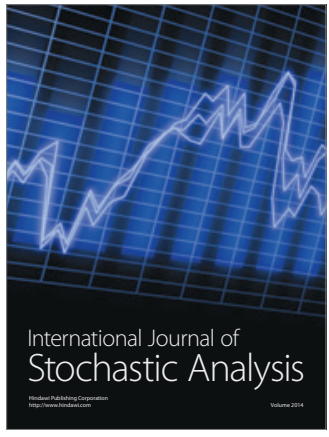

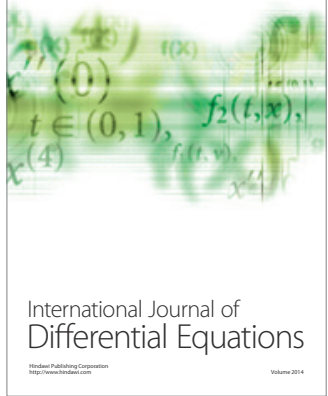
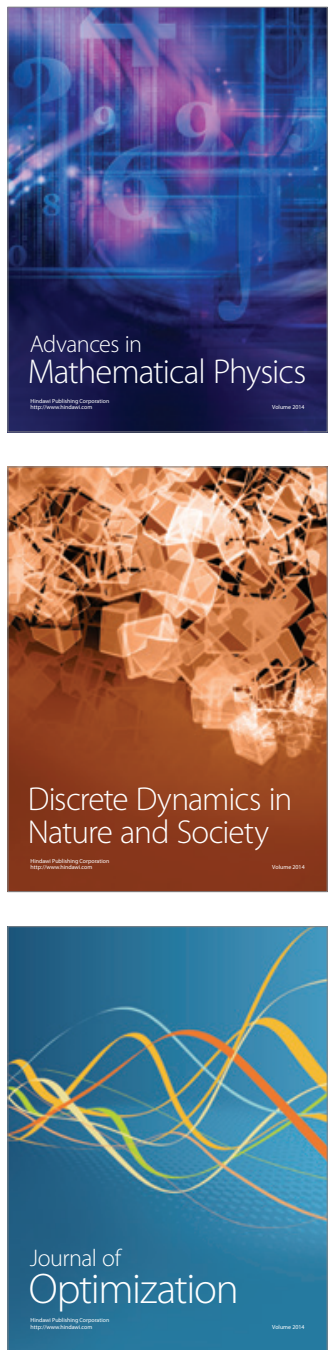